Success Rate and Technical Quality of Home

Polysomnography With Self-Applicable Electrode Set in

Subjects With Possible Sleep Bruxism

\title{
Miettinen, Tomi
}

2018-07

Miettinen , T , Myllymaa , K, Westeren-Punnonen , S , Ahlberg , J , Hukkanen , T , Töyräs , J , Lappalainen, R, Mervaala , E, Sipilä, K \& Myllymaa, S 2018, ' Success Rate and

Technical Quality of Home Polysomnography With Self-Applicable Electrode Set in Subjects With Possible Sleep Bruxism ' , IEEE Journal of Biomedical and Health Informatics, vol. 22 , no. 4 , pp. 1124-1132 . https://doi.org/10.1109/JBHI.2017.2741522

http://hdl.handle.net/10138/310899

https://doi.org/10.1109/JBHI.2017.2741522

unspecified

acceptedVersion

Downloaded from Helda, University of Helsinki institutional repository.

This is an electronic reprint of the original article.

This reprint may differ from the original in pagination and typographic detail.

Please cite the original version. 


\title{
Success Rate and Technical Quality of Home Polysomnography with Self-Applicable Electrode Set in Subjects with Possible Sleep Bruxism
}

\author{
Tomi Miettinen, Katja Myllymaa, Susanna Westeren-Punnonen, Jari Ahlberg, Taina Hukkanen, Juha \\ Töyräs, Reijo Lappalainen, Esa Mervaala, Kirsi Sipilä, Sami Myllymaa
}

\begin{abstract}
Using sleep laboratory polysomnography (PSG) is restricted for the diagnosis of only the most severe sleep disorders due to its low availability and high cost. Home PSG is more affordable, but applying conventional electroencephalography (EEG) electrodes increases its overall complexity and lowers the availability. Simple, self-administered single-channel EEG monitors on the other hand suffer from poor reliability. In this study, we aimed to quantify the reliability of self-administrated home PSG recordings conducted with a newly designed ambulatory electrode set (AES) that enables multi-channel EEG, electrooculography, electromyography and electrocardiography recordings. We assessed the sleep study success rate and technical quality of the recordings performed in subjects with possible sleep bruxism (SB). Thirty-two females and five males aged 39.6 \pm 11.6 years $($ mean \pm SD) with self-reported $S B$ were recruited in the study. Self-administrated home PSG recordings with two AES designs were conducted ( $n=19$ and 21$)$. The technical quality
\end{abstract}

This manuscript was submitted for review February 15th, 2017 and resubmitted after revisions July 3rd, 2017 and August 11th, 2017. This work was financially supported by The Finnish Funding Agency for Technology and Innovation project of 40047/14 ('Bruxism'), and by grants from the Research Committee of Kuopio University Hospital Catchment Area for the State Research Funding (projects 5041739, 5041748 and 5041761) and Ulla Tuominen Foundation. T. Miettinen was supported by Instrumentarium Science Foundation and North Savo Regional fund of Finnish Cultural Foundation.

T. Miettinen, K. Myllymaa, S. Westeren-Punnonen, T. Hukkanen, J. Töyräs and E. Mervaala are with Department of Clinical Neurophysiology, Kuopio University Hospital, Kuopio 70029, Finland. T. Miettinen, K. Myllymaa and J. Töyräs are also with the Department of Applied Physics, University of Eastern Finland, Kuopio 70211, Finland. T. Miettinen is also with Institute of Dentistry, University of Eastern Finland, Kuopio 70211, Finland. E. Mervaala is also with the Institute of Clinical Medicine, University of Eastern Finland, Kuopio 70211, Finland. (e-mails tomi.miettinen@uef.fi, katja.myllymaa@kuh.fi, susanna.westeren-punnonen@kuh.fi, taina.hukkanen @ kuh.fi, juha.toyras@uef.fi and esa.mervaala@kuh.fi)

J. Ahlberg is with the Department of Oral and Maxillofacial Diseases, University of Helsinki, Helsinki 00014, Finland (e-mail jari.ahlberg@ @elsinki.fi).

R. Lappalainen and S. Myllymaa are with the Department of Applied Physics, University of Eastern Finland, Kuopio 70211, Finland. S. Myllymaa is and also with Institute of Dentistry, University of Eastern Finland, Kuopio 70211, Finland. (e-mails reijo.lappalainen@uef.fi and sami.myllymaa@uef.fi)

K. Sipilä is with the Institute of Dentistry, University of Eastern Finland, Kuopio 70211, Finland and also with Department of Oral and Maxillofacial Diseases, Kuopio University Hospital, Kuopio 70029, Finland and Oral and Maxillofacial Department, Oulu University Hospital, Oulu 90029, Finland. (email kirsi.sipila@uef.fi) of the recordings was graded based on the proportion of interpretable data. Technical failure rate for AES (both designs) was $5 \%$ and SB was scorable for $96.9 \%$ of all recorded data. Only one recording failed due to mistakes in self-applying the AES. We found that the proportion of good quality selfadministrated EEG recordings is significantly higher when multiple channels are used compared to using a single channel. Sleep study success rates and proportion of recordings with high quality interpretable data from EEG channels of AES were comparable to that of conventional home PSG. Self-applicable AES has potential to become a reliable tool for widely available home PSG.

Index Terms - Biomedical electrodes, electroencephalography, electromyography, printed circuits, thick film sensors.

\section{INTRODUCTION}

Qleep disorders cause significant harm on the health and $\checkmark$ well-being for millions of people worldwide and are costly for the healthcare system [1]-[4]. Currently, sleep laboratory polysomnography (PSG) is the gold standard method for diagnosing various sleep disorders, such as obstructive sleep apnea and narcolepsy [5]. However, it is considered to be cumbersome and expensive. In addition, sleep laboratory facilities are not easily available everywhere, especially in rural areas. Due to high costs and limited availability, use of sleep laboratory PSG has usually been prioritized mainly for the diagnosis of the clinically most severe sleep disorders.

PSG at home setting with its lower costs [6] may be more feasible compared to sleep laboratory PSG. However, the full diagnostic potential of home PSG is limited by the conventional electroencephalography (EEG) electrodes that need to be applied by a technician. The complexity of electrode appliance limits the availability of full home PSG only to be provided by facilities that have personnel with skills in EEG electrode appliance. Recently, devices with simple single channel EEG electrodes have been introduced with the possibility of patient self-appliance [7]-[9]. However, in recordings with a single EEG channel there is a high risk of total failure of the recording due to detachment, misplacement or failure of an EEG electrode [7], [9]. 
We have recently introduced a silver ink screen-printed and hydrogel-coated ambulatory electrode set [10] (AES) for emergency assessment of EEG [11]. AES is easy and quick to apply and in comparison to sleep laboratory PSG it has been shown to be suitable for sleep staging and determination of total sleep time [12] as well as detecting sleep bruxism events [13]. To our best knowledge, there are currently no other multi-channel EEG, EOG and EMG electrode sets for sleep monitoring that could be applied by patients themselves. AES has a large range of applications in the diagnostics of sleep disorders. However, originally it has not been designed to be used at home or tested in a home setting. We hypothesize that the AES could be developed to be easily self-applicable by patients, enabling more available, reliable and good quality home PSG recordings. We also hypothesize that using multichannel EEG, EOG and EMG in self-applied home PSG enhances the success rates of the recordings compared to single-channel recordings. In this study, we specifically tested the success rate and the technical quality of home PSG recordings made with AES in subjects with possible sleep bruxism (SB).

SB is a sleep-related movement disorder that is currently underdiagnosed due to the limited availability of sleep laboratory PSG required to make a definite diagnosis [14]. Self-reported SB is considered sufficient only for a 'possible' diagnosis [14]. It needs to be confirmed with a clinical examination ('probable' diagnosis) and with PSG ('definite' diagnosis) [14]. PSG diagnosis of SB is based on the rhythmic masticatory muscle activity (RMMA) index, determined as the number of RMMA events per total sleep time [15]. The current commonly used RMMA index cut-off value for positive $\mathrm{SB}$ diagnosis is $\geq 2$, with diagnosis additionally requiring positive tooth-grinding history or confirmed grinding in audio recording [15].

Accurate determination of RMMA index requires recording of a combination of different biosignals. Masseter (and/or temporal) muscle electromyography (mass-EMG) is needed for the detection of RMMA events. RMMA events consist of EMG bursts that are scored on masseter or temporalis EMG channels when the EMG amplitude rises to a level of at least $10 \%$ of maximum voluntary clenching activity [15] or twice the baseline amplitude [16] for a minimum duration of $0.25 \mathrm{~s}$. Recognition of the subtypes of RMMA (phasic, tonic or mixed) is important, since they are associated with different clinical signs and symptoms of SB [17]. A phasic RMMA event consists of at least three bursts lasting $0.25 \mathrm{~s}$ to $2 \mathrm{~s}$ with maximum of $2 \mathrm{~s}$ in between [15]. A tonic RMMA event corresponds to a burst with a duration longer than $2 \mathrm{~s}$ and a mixed RMMA event includes both tonic and phasic bursts [15]. Audio-video recordings are needed for distinguishing RMMA events from other orofacial or muscular activities (e.g. yawning, swallowing or changing position) to prevent possible overestimation of the number of RMMA events [18],[19] and to confirm SB diagnosis with the presence of teeth-grinding sounds [15]. In addition, EEG, electrooculography (EOG) and submental EMG (sub-EMG) recordings are needed for sleep staging, determination of total sleep time and distinguishing SB from bruxism during wakefulness [15]. Portable EMG devices have been developed for simple diagnostics of SB [20] -[26], but their diagnostic accuracy compared to sleep laboratory PSG is questionable due to the lack of EEG based sleep staging and ability to distinguish between SB and other facial or muscular activities.

Currently, practitioners of sleep medicine make the analysis for the diagnosis subjectively by identifying sleep stages and events based on the recorded signals manually. However, if available and reliable, automatic sleep stage and event detection would be less time-consuming. Micro-arousals precede most RMMA events [27], which makes an increase in EEG and electrocardiography (ECG) activity potential markers for more accurate automatic RMMA event recognition. Castroflorio et al. have shown that automatic RMMA event recognition based on detecting increased heart rate before RMMA events when recorded with portable device leads to high RMMA index agreement with subjectively analyzed portable PSG [25]. High quality recording techniques are the best way to prevent erroneous results in automatic analyses [28]. Thus, a detailed analysis of the quality of the signals and the sources for avoidable technical artifacts is a prerequisite for the estimation of the potential of using automatic analysis methods.

The aim of this study was to quantify the reliability of the self-applied home PSG recordings conducted with a newly designed multi-channel AES in subjects with self-reported SB. The reliability was assessed by the means of success rate and technical quality of the recordings and signals. Detailed reasons for signal losses were also assessed as well as electrical impedances of the electrodes and subjective quality of applying the electrodes.

\section{METHODS}

\section{A. Subjects}

Recordings for the assessment of the reliability of AES in home PSG for SB diagnostics were conducted in two stages. Eighteen female and one male voluntary subjects aged $40.9 \pm$ 11.9 years (mean $\pm \mathrm{SD}$ ) were recruited for the recordings conducted in stage 1 . In stage 2 , sixteen females and five males aged $38.4 \pm 11.7$ years were recruited, of which two females and one male participated also in stage 1 . All subjects in stage 1 and eleven subjects in stage 2 were recruited from volunteers that responded to an open call for test subjects posted in intrawebs of Kuopio University Hospital (KUH, Kuopio, Finland) and University of Eastern Finland (UEF, Kuopio, Finland). Ten subjects in stage 2 were recruited by author J.A. from volunteered patients in a dental clinic of the Unit of Specialized Oral Care in the Metropolitan Area and Kirkkonummi (Helsinki, Finland).

Inclusion criteria for all subjects was self-reported SB that occurred at least one night per week. Informed consent was obtained from all individual participants included in the study. The study protocol was reviewed by the Research Ethics Committee of the Hospital District of Northern Savo, Kuopio, Finland (favorable opinion: 34/2013) and a permission was obtained from the National Supervisory Authority for Welfare and Health (Valvira, 220/2013).

\section{B. Electrode set and its development stages}

For stage 1 of the study, a new design of the ambulatory 

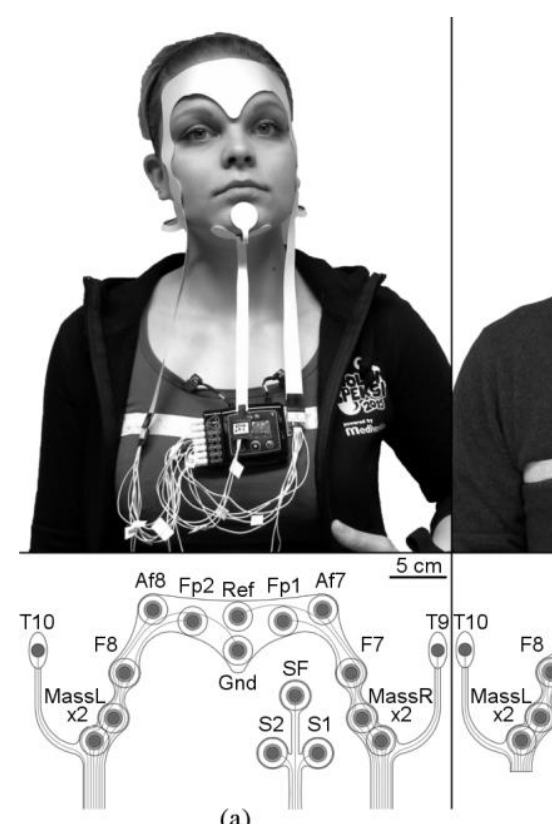

(a)

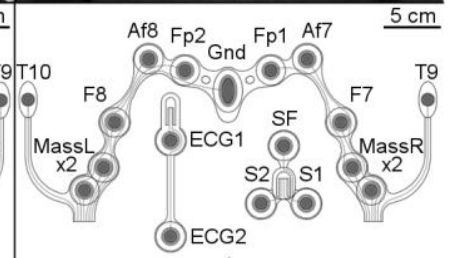

(b)

Fig. 1. Ambulatory electrode sets. Photographs of the screen printed ambulatory electrode sets (AES) and other sensors and devices used in the recordings and diagrams of corresponding electrode positions used in (a) stage 1 of the study (AES-1) and in (b) stage 2 of the study (AES-2). Separate ECG monitor applied in stage 1 and ECG electrodes applied in stage 2 are not visible in the photographs.

electrode set (AES-1, Fig. 1a), enabling easier self-application was developed from the original emergency EEG electrode set. The materials and the layer structure of the new AES- 1 are similar to the original set and described in detail elsewhere [10]. All materials in contact with the skin are approved for medical use.

AES-1 consists of two parts (Fig. 1a). The larger part had four EEG electrodes (Af8, Fp2, Fp1, Af7), one ground electrode (Gnd) and one extra reference electrode (Ref) positioned on the forehead, two electrodes for EOG positioned in F8 and F7, four masseter EMG (mass-EMG) electrodes (MassL, MassR, two bilaterally) and two mastoidal reference electrodes (T10, T9). The smaller part of AES-1 consisted of three submental (sub-EMG) electrodes (S1, S2, SF).

Based on the technical quality of the data and subject questionnaire results of stage 1 recordings, an improved electrode set (AES-2) was developed and tested in stage 2 (Fig. 1b). EEG electrodes Af8 and Af7 were repositioned 5 $\mathrm{mm}$ and Fp2 and Fp1 $2 \mathrm{~mm}$ closer to the vertical centerline of the face to prevent applying the electrodes on top of the hair. Ref electrode was removed as unused and the ground electrode Gnd was made larger for improved electrical contact with skin. Laser-cut outline of the polyester film was changed to cover less skin and thus reduce sweating. The long polyester strips extending from the facial area to chest area were found to be uncomfortable and were replaced with longer cables which were attached to sockets near the mass-EMG electrodes. ECG electrode strip was introduced in AES-2, with ECG2 electrode to be positioned on top of heart and ECG1 electrode in $45^{\circ}$ angle to horizontal line and to be placed approximately on the middle vertical line of the chest.

\section{Data acquisition}

All home PSG recordings were conducted by using Nox A1 portable polysomnography system (Nox Medical, Reykjavík, Iceland) with respiratory inductance plethysmography (RIP) chest band that also holds the recording device on the chest. Nox A1 was supplemented in stage 1 with an ECG monitor eMotion Faros $180^{\circ}$ (Mega Electronics Ltd, Kuopio, Finland) with bipolar derivation, one electrode positioned on top of heart and the other one horizontally on the opposite side of the chest.

AES and rest of the equipment needed for home PSG recordings were given and introduced to subjects the same day the PSG recording was to take place. A skilled technician (in stages 1 and 2, studies conducted from the Department of Clinical Neurophysiology of KUH) or a dentist having had a two-hour introduction to the used PSG equipment (in stage 2, studies conducted from the dental clinic) instructed subjects how to apply the AES and other PSG equipment by themselves before going to sleep. Written instructions with detailed illustrations were also given to subjects to take home for referring to when applying the equipment. Subjects were asked to fill in a set of questionnaires, which included questions concerning the time of going to sleep, falling asleep and waking up, ease of applying the AES and other PSG equipment on scale from $1=$ easy to $10=$ difficult, as well as problems encountered during the night with the equipment.

The PSG recording device was programmed by a technician or the dentist to automatically turn on and record for a pre-set duration of time, depending on subject's approximation for the time of going to sleep and waking up. Before going to sleep, subjects were told to prepare the skin by wiping the electrode attachment sites with a disposable disinfectant wipe. After the recordings, subjects returned all recording devices to the facility they were given from.

The recording montage in stage 1 consisted of four EEG derivations (Fp1-T10, Fp2-T9, Af8-T9, Af7-T10), two EOG derivations (F8-T9, F7-T10), two sub-EMG derivations (S1$\mathrm{SF}$ and S2-SF) and two bipolar mass-EMG derivations from MassL and MassR electrodes. ECG was recorded with the separate bipolar ECG monitor in stage 1, which was temporally synchronized with the PSG recording device. PSG device had embedded activity, position and audio recordings as well as recording of respiratory movements with a RIP band. The PSG recording device measured the electrode impedances through the night. Recording montage in stage 2 was similar, except for the separate ECG device that was replaced with ECG electrodes of AES-2 (ECG recorded with the Nox A1 recording device).

\section{Analysis}

Technical quality of scored recordings was graded by the primary author (T.M.) according to a modified version of the grading system introduced by Redline et al. [29], originally developed for unattended PSG conducted to diagnose sleepdisordered breathing. In this system, all channels were given a quality grade based on duration of interpretable signal, which formed the basis for the overall grading of each study. The 
applied grading system was modified by replacing the channels needed in diagnostics of sleep-disordered breathing with ones recorded to diagnose SB (Table I). The subjective dichotomization of signal quality as interpretable or noninterpretable was made channel-wise by marking the noninterpretable or artifact-ridden periods of the signal in the recordings. The channels were chosen as the ones needed in determining the RMMA index: detecting RMMA events and distinguishing them from other events (Fig. 2) or determining total sleep time. Reasons for signal losses or technical artifacts in all channels were analyzed from the recordings during the grading. Subjects' accounts on encountered problems during the study were used as supplementary material in the analysis.

Proportions of total scorable data to total recording time were also determined. Recorded data were considered scorable for SB at any time point, if it included the following: at least one interpretable EEG channel for the assessment of subject being in sleep or awake; one interpretable mass-EMG channel for the recognition of bruxism episodes; and audio recording for distinguishing SB from other orofacial or muscular activities. Total recording time was determined as the time between subject going to bed and subject waking up. The time

TABLE I

TECHNICAL QUALITY GRADING SYSTEM OF THE RECORDINGS

\begin{tabular}{|c|c|}
\hline Quality Grade & Requirements for the Grade \\
\hline Outstanding & All channels good for $\geq 6 \mathrm{~h}$ \\
\hline Excellent & $\begin{array}{l}\text { At least } 2 \times E E G, 1 \times \text { EOG, sub-EMG, mass-EMG and } \\
\text { audio, ECG good for } \geq 5 \mathrm{~h}\end{array}$ \\
\hline Very Good & $\begin{array}{l}\text { At least } 1 \mathrm{xEEG}, \mathrm{EOG} \text {, sub-EMG, mass-EMG and } \\
\text { audio good for } \geq 5 \mathrm{~h}\end{array}$ \\
\hline Good & At least $1 \mathrm{xEEG}$, mass-EMG and audio good for $\geq 5 \mathrm{~h}$ \\
\hline Fair & $\begin{array}{l}\text { At least } 1 \mathrm{xEEG} \text {, mass-EMG and audio good for } \leq 5 \mathrm{~h} \\
\text { but } \geq 4 \mathrm{~h}\end{array}$ \\
\hline Poor & $\begin{array}{l}\text { EEG, mass-EMG and audio channels contain } \leq 4 \mathrm{~h} \text { of } \\
\text { interpretable data }\end{array}$ \\
\hline Unsatisfactory & No usable data, less than 2 hours on all channels \\
\hline
\end{tabular}

Recordings rated poor or unsatisfactory were considered as failed recordings. Modified from [29]. EEG = electroencephalography, EOG = electrooculography, sub-EMG = submental electromyography, mass-EMG = masseter electromyography.

RMMA event detection

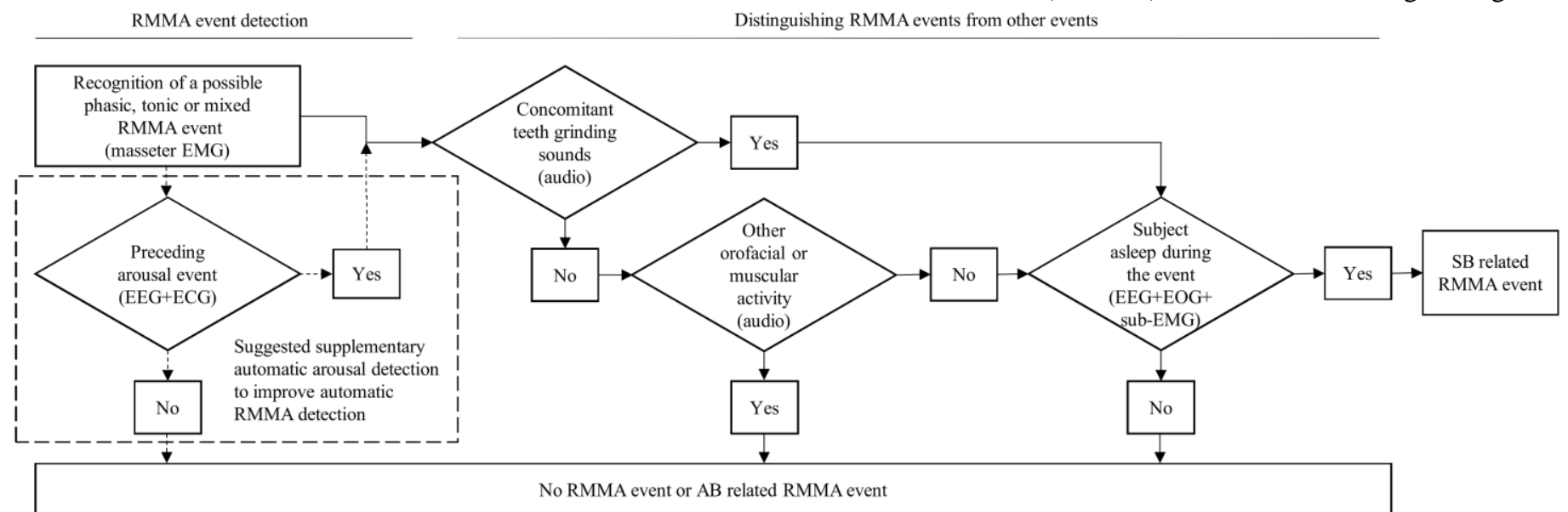

Fig 2. Flow chart for detecting RMMA events and distinguishing RMMA events from other events with ambulatory electrode set and PSG recording equipment used in this study. The channels that are used in the decision making are in parenthesis inside the boxes. Subjective evaluation of detected RMMA events includes listening to the audio playback and scoring the sleep stages to distinguish the type of the event. Inside the dashed box on the left side of the figure is the suggested additional step to enhance the accuracy of the initial RMMA event detection, if the automatic RMMA detection methods would be implemented.

$\mathrm{AB}=$ awake bruxism, $\mathrm{ECG}$ = electrocardiography, $\mathrm{EEG}=$ electroencephalography, $\mathrm{EOG}=$ electrooculography, sub-EMG = submental electromyography, EMG = electromyography, $\mathrm{PSG}=$ polysomnography, $\mathrm{RMMA}=$ rhythmic masticatory muscle activity, $\mathrm{SB}=$ sleep bruxism. of subject going to bed (starting point of analysis) was set at the earliest time point when the continuous movement activity in actigraph and other channels seized before falling asleep close to the subject's reported time of going to sleep. The ending point of the analysis was set to the time point of subject waking up. The time points of falling asleep and waking up were determined based on sleep stage scoring of EEG. In stage 1, sleep stages were scored by an experienced KUH clinical neurophysiologist (S.W-P.). In stage 2, scoring was made by an expert technologist (Oivauni Oy, Kuopio, Finland), certified by European Sleep Research Society. All sleep scorings were carried out in 30 second epochs with soxturnal software (Nox Medical, Reykjavík, Iceland) cording to AASM guidelines [16]. The rule for scoring N1 in subjects who do not create alpha rhythm was used, the recording montage did not include occipital rhythm [16].

Electrical impedances of all working electrodes recorded by the PSG device were analyzed by taking a 60 second mean impedance value every hour from the time of going to sleep. The first impedance value was taken from the time point of subject going to bed and rest of the impedance data points every full hour after this.

Subject questionnaire results concerning the ease of applying AES and other PSG equipment were statistically analyzed using SPSS software (version 21.0; SPSS, Chicago, IL, USA). Shapiro-Wilk test was used to evaluate whether the data sets follow normal distribution. Mann-Whitney U-test was used for the comparison of mean values of the ease of applying the AES and other PSG equipment. Threshold for statistical significance was $p=0.05$.

\section{RESULTS}

Out of the forty sleep studies conducted, the quality of thirty-six recordings $(90 \%)$ was graded "good" or better, and two recordings (5\%, one recording in both stages) were considered as failed (Table II). The failed recording in stage 1 
TABLE II

TECHNICAL QUALITY GRADES OF THE RECORDINGS

\begin{tabular}{|c|c|c|c|}
\hline Quality Grade & All Recordings & Stage 1 & Stage 2 \\
\hline Outstanding & $15(37.5 \%)$ & $7(37 \%)$ & $8(38 \%)$ \\
\hline \multirow[t]{5}{*}{ Excellent } & $17(42.5 \%)$ & $10(53 \%)$ & $7(33 \%)$ \\
\hline & & EEG or EOG sweat artifact $2(11 \%)$ & TRT less than six hours, $2(9.5 \%)$ \\
\hline & & Bad contact in submental reference electrode, 1 (5.3\%) & Bad contact in masseter-EMG electrode, 1 (4.8\%) \\
\hline & & & Recording ended prematurely, $1(4.8 \%)$ \\
\hline & & & EEG or EOG sweat artifact, $1(4.8 \%)$ \\
\hline Good & & - & Submental EMG electrodes' cable detachment, 4 (19.0\%) \\
\hline \multirow[t]{2}{*}{ Fair } & $2(5 \%)$ & $1(5 \%)$ & $1(5 \%)$ \\
\hline & & Bad contact in EEG reference electrodes, 1 (5.3\%) & Submental EMG electrodes' cable detachment, 1 (4.8\%) \\
\hline \multirow[t]{2}{*}{ Poor } & $1(2.5 \%)$ & $1(5 \%)$ & $0(0.0 \%)$ \\
\hline & & Recording device malfunction, 1 (5.3\%) & - \\
\hline \multirow[t]{2}{*}{ Unsatisfactory } & $1(2.5 \%)$ & $0(0.0 \%)$ & $1(5 \%)$ \\
\hline & & - & Electrode covers were not removed, 1 (4.8\%) \\
\hline
\end{tabular}

Number and proportion of recordings with corresponding technical quality grades and reasons for grades lower than "outstanding" for recordings conducted in stage 1 and stage 2 of the study with the new designs of the ambulatory electrode sets. Recordings rated poor or unsatisfactory were considered as failed recordings.

$\mathrm{EEG}=$ electroencephalography, $\mathrm{EOG}=$ electrooculography, $\mathrm{EMG}=$ electromyography, $\mathrm{TRT}=$ Total recording time.

was due to a partial loss of all recorded data, caused by a malfunction of the recording device. The failed recording in stage 2 was due to a mistake in electrode attachment as the subject did not remove electrode covers before going to bed. When full data losses caused by the recording device were excluded, SB could be scored for $96.9 \%$ of all TRT.

There were various reasons for data losses causing study quality being other than "outstanding" (Table II). However, in most of the cases these problems were restricted only to a small proportion of the electrodes at a time. At least one channel (any single channel) in all signal groups (EEG, EOG, sub-EMG, mass-EMG, ECG) had the highest quality grade "A" (more than $95 \%$ of interpretable data) in $84 \%$ or more of the recordings made in stage 1 (Table III). Similarly, in recordings made in stage 2 the proportion of recordings with the highest quality grade " $\mathrm{A}$ " in any channel within the signal group was almost at the same level ( $81 \%$ or more) with the exception of sub-EMG signal group that had significantly more problems on both channels at the same time. In single channels, proportions of the recordings with quality grade " $\mathrm{A}$ " were generally lower compared to the situation where proportion of interpretable data is taken from any channel.

A more detailed description of the reasons behind signal losses is presented in Table IV. In $37 \%$ (stage 1) and $24 \%$ (stage 2) of the recordings, at least one electrode had initial bad contact that caused signal loss that started from the beginning of the recording. Most of the initial signal quality problems were in EEG channels Af8-T9 and Af7-T10, especially in stage 1 recordings. According to subjects' feedback, in some cases electrodes Af8 and Af7 had to be applied too close to hairline due to their fixed positions in the electrode set. Changing the positions of Af8 and Af7 electrodes further away from the hairline led to smaller proportion of lost signal in these electrodes due to bad electrode contact in stage $2(4.5 \%$ and $4.1 \%$, respectively) compared to stage 1 (10.7\% and $28.9 \%$, respectively). In stage 2, the highest proportion of lost signal was in sub-EMG signals due to cable detachment. In addition, mistakenly set wrong recording times and increased sweat artifact were an issue in stage 2 recordings. Out of the 21 recordings in stage 2, temporally $76.3 \%$ of all signal losses happened in ten recordings made in the dental clinic.

TABLE III

SIGNAL QUALITY GRADES IN THE RECORDINGS

\begin{tabular}{|c|c|c|c|c|c|c|c|c|c|c|c|c|c|c|c|}
\hline Quality Grade & $\begin{array}{c}\text { Fp2- } \\
\text { T9 }\end{array}$ & $\begin{array}{l}\text { Fp1- } \\
\text { T10 }\end{array}$ & $\begin{array}{c}\text { Af8- } \\
\text { T9 }\end{array}$ & $\begin{array}{l}\text { Af7- } \\
\text { T10 }\end{array}$ & $\begin{array}{l}\text { Any } \\
\text { EEG }\end{array}$ & $\begin{array}{l}\text { F8- } \\
\text { T9 }\end{array}$ & $\begin{array}{l}\text { F7- } \\
\text { T10 }\end{array}$ & $\begin{array}{l}\text { Any } \\
\text { EOG }\end{array}$ & $\begin{array}{l}\text { S1- } \\
\text { SF }\end{array}$ & $\begin{array}{l}\text { S2- } \\
\text { SF }\end{array}$ & $\begin{array}{l}\text { Any } \\
\text { sub- } \\
\text { EMG }\end{array}$ & MassL & MassR & $\begin{array}{c}\text { Any } \\
\text { mass- } \\
\text { EMG }\end{array}$ & ECG \\
\hline \multicolumn{16}{|l|}{ Stage 1} \\
\hline$A: 100 \%-95 \%$ & $79 \%$ & $69 \%$ & $68 \%$ & $42 \%$ & $84 \%$ & $84 \%$ & $74 \%$ & $84 \%$ & $68 \%$ & $84 \%$ & $90 \%$ & $79 \%$ & $84 \%$ & $90 \%$ & $90 \%$ \\
\hline B: $94 \%-75 \%$ & $11 \%$ & $26 \%$ & $11 \%$ & $21 \%$ & $11 \%$ & $11 \%$ & $21 \%$ & $11 \%$ & $16 \%$ & $11 \%$ & $10 \%$ & $11 \%$ & $16 \%$ & $10 \%$ & $10 \%$ \\
\hline$C: 74 \%-50 \%$ & $10 \%$ & $5 \%$ & $11 \%$ & $5 \%$ & $5 \%$ & $5 \%$ & $5 \%$ & $5 \%$ & $16 \%$ & $5 \%$ & - & $5 \%$ & - & - & - \\
\hline$D:<50 \%$ & - & - & $10 \%$ & $32 \%$ & - & - & - & & & & - & $5 \%$ & - & - & - \\
\hline \multicolumn{16}{|l|}{ Stage 2} \\
\hline A: $100 \%-95 \%$ & $71 \%$ & $76 \%$ & $57 \%$ & $52 \%$ & $81 \%$ & $81 \%$ & $76 \%$ & $81 \%$ & $67 \%$ & $67 \%$ & $67 \%$ & $85 \%$ & $86 \%$ & $90 \%$ & $90 \%$ \\
\hline B: $94 \%-75 \%$ & $10 \%$ & $14 \%$ & $29 \%$ & $24 \%$ & $9 \%$ & $9 \%$ & $10 \%$ & $9 \%$ & - & - & - & $5 \%$ & - & $5 \%$ & $5 \%$ \\
\hline C: $74 \%-50 \%$ & $14 \%$ & $5 \%$ & $5 \%$ & $14 \%$ & $5 \%$ & $5 \%$ & $9 \%$ & $5 \%$ & $9 \%$ & $9 \%$ & $9 \%$ & $5 \%$ & - & - & - \\
\hline$D:<50 \%$ & $5 \%$ & $9 \%$ & $9 \%$ & $10 \%$ & $5 \%$ & $5 \%$ & $5 \%$ & $5 \%$ & $24 \%$ & $24 \%$ & $24 \%$ & $5 \%$ & $14 \%$ & $5 \%$ & $5 \%$ \\
\hline
\end{tabular}

Signal quality of the recordings with the new designs of the ambulatory electrode set in stage 1 and stage 2 of the study. Signal quality is expressed channelwise as proportion of recordings with interpretable data lasting 100\% - 95\%, 94\% - 75\%, 74\% - 50\% and less than 50 \% of the recorded signal. Full data losses caused by recording device malfunction or problems in setting up the recording device are excluded from this table. Audio was distortion free in all recordings.

ECG = electrocardiography, EEG = electroencephalography, EOG = electrooculography, sub-EMG = submental EMG, mass-EMG = masseter EMG. 
TABLE IV

REASONS FOR SIGNAL LOSSES IN THE RECORDINGS.

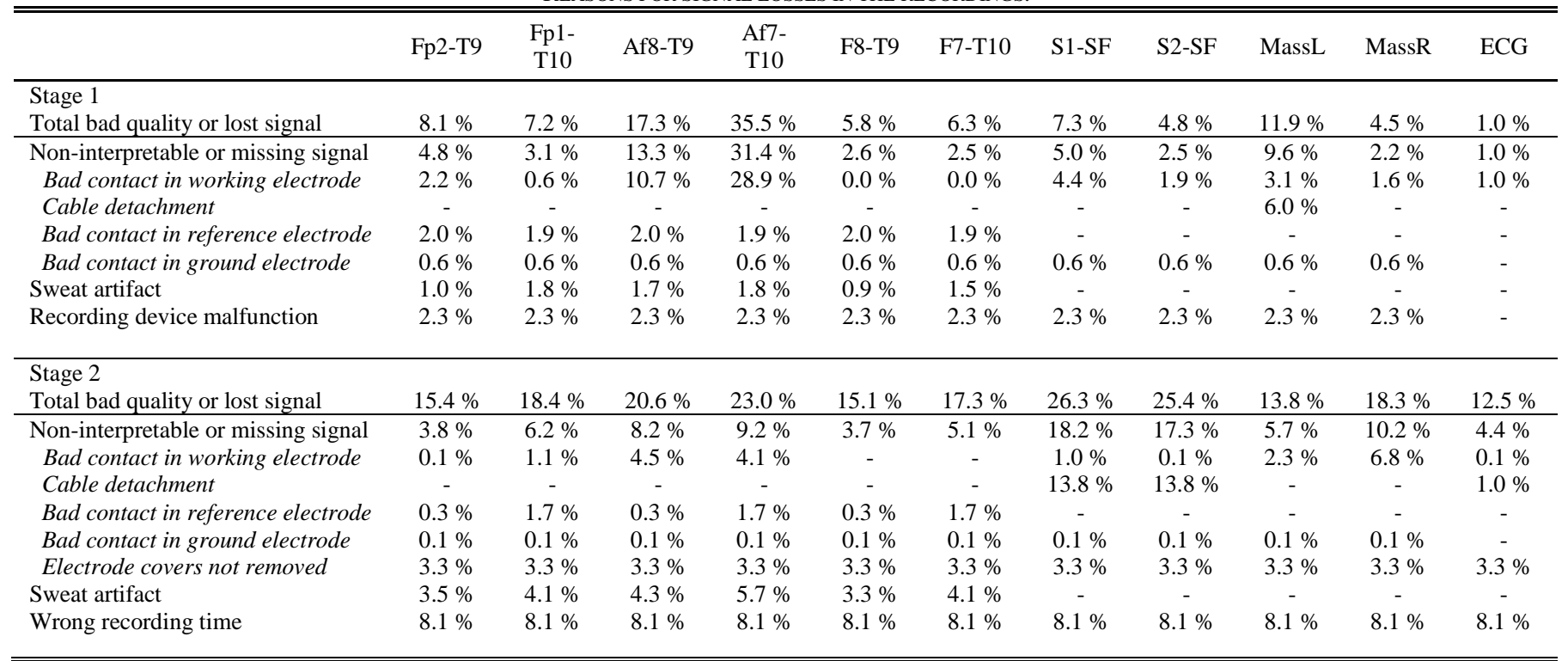

Reasons for signal losses in the recordings with new designs of the ambulatory electrode set in stage 1 and stage 2 of the study. Data are expressed as ratio of total duration of lost signals to sum of total recording times in all recordings.

Electrical impedances of electrodes were below $75 \mathrm{k} \Omega$ in approximately $90 \%$ of the measured data points in stage 1 with AES-1, except of electrodes Af8 and Af7 (Table V). These two electrodes had impedance values higher than 300 $\mathrm{k} \Omega$ in $25 \%$ and $42 \%$ of data points, usually at device maximum value of $328 \mathrm{k} \Omega$. Repositioning the electrodes in stage 2 lowered the electrical impedances. Generally, electrical contact of the electrodes on skin showed no significant decay during recordings in terms of electrical impedance and bad electrical contact was most often present

TABLE V

ELECTRICAL IMPEDANCES OF THE WORKING ELECTRODES OF AMBULATORY ELECTRODE SET.

\begin{tabular}{|c|c|c|c|c|c|c|c|c|c|c|c|}
\hline$Z(\mathrm{k} \Omega)$ & Fp2 & Fp1 & Af8 & Af7 & F8 & F7 & S1 & S2 & SF & MassL & MassR \\
\hline \multicolumn{12}{|l|}{ Stage 1} \\
\hline $0-10$ & $29 \%$ & $61 \%$ & $8 \%$ & $14 \%$ & $24 \%$ & $68 \%$ & $29 \%$ & $48 \%$ & $33 \%$ & $0 \%$ & $0 \%$ \\
\hline $10-20$ & $30 \%$ & $16 \%$ & $9 \%$ & $20 \%$ & $32 \%$ & $12 \%$ & $66 \%$ & $46 \%$ & $58 \%$ & $18 \%$ & $14 \%$ \\
\hline $20-30$ & $12 \%$ & $9 \%$ & $15 \%$ & $8 \%$ & $19 \%$ & $9 \%$ & $3 \%$ & $2 \%$ & $5 \%$ & $43 \%$ & $61 \%$ \\
\hline $30-40$ & $10 \%$ & $2 \%$ & $20 \%$ & $5 \%$ & $10 \%$ & $0 \%$ & $0 \%$ & $3 \%$ & $0 \%$ & $14 \%$ & $12 \%$ \\
\hline $40-50$ & $3 \%$ & $1 \%$ & $5 \%$ & $0 \%$ & $3 \%$ & $0 \%$ & $0 \%$ & $1 \%$ & $0 \%$ & $14 \%$ & $11 \%$ \\
\hline $50-75$ & $2 \%$ & $1 \%$ & $10 \%$ & $3 \%$ & $2 \%$ & $1 \%$ & $0 \%$ & $0 \%$ & $0 \%$ & $4 \%$ & $1 \%$ \\
\hline $75-100$ & $1 \%$ & $2 \%$ & $2 \%$ & $1 \%$ & $1 \%$ & $1 \%$ & $0 \%$ & $0 \%$ & $0 \%$ & $0 \%$ & $0 \%$ \\
\hline $100-200$ & $2 \%$ & $0 \%$ & $2 \%$ & $5 \%$ & $1 \%$ & $1 \%$ & $0 \%$ & $0 \%$ & $3 \%$ & $0 \%$ & $0 \%$ \\
\hline $200-300$ & $3 \%$ & $1 \%$ & $3 \%$ & $1 \%$ & $2 \%$ & $3 \%$ & $0 \%$ & $0 \%$ & $0 \%$ & $0 \%$ & $0 \%$ \\
\hline$>300$ & $7 \%$ & $7 \%$ & $25 \%$ & $42 \%$ & $6 \%$ & $5 \%$ & $3 \%$ & $1 \%$ & $0 \%$ & $7 \%$ & $0 \%$ \\
\hline \multicolumn{12}{|l|}{ Stage 2} \\
\hline $0-10$ & $35 \%$ & $35 \%$ & $16 \%$ & $13 \%$ & $37 \%$ & $42 \%$ & $39 \%$ & $37 \%$ & $33 \%$ & $0 \%$ & $4 \%$ \\
\hline $10-20$ & $32 \%$ & $36 \%$ & $31 \%$ & $30 \%$ & $41 \%$ & $35 \%$ & $42 \%$ & $47 \%$ & $47 \%$ & $19 \%$ & $13 \%$ \\
\hline $20-30$ & $13 \%$ & $17 \%$ & $19 \%$ & $11 \%$ & $10 \%$ & $11 \%$ & $2 \%$ & $2 \%$ & $2 \%$ & $42 \%$ & $42 \%$ \\
\hline $30-40$ & $7 \%$ & $2 \%$ & $5 \%$ & $12 \%$ & $3 \%$ & $2 \%$ & $9 \%$ & $4 \%$ & $6 \%$ & $27 \%$ & $31 \%$ \\
\hline $40-50$ & $1 \%$ & $2 \%$ & $2 \%$ & $7 \%$ & $1 \%$ & $3 \%$ & $0 \%$ & $0 \%$ & $0 \%$ & $5 \%$ & $0 \%$ \\
\hline $50-75$ & $4 \%$ & $1 \%$ & $12 \%$ & $9 \%$ & $2 \%$ & $1 \%$ & $2 \%$ & $0 \%$ & $0 \%$ & $2 \%$ & $1 \%$ \\
\hline $75-100$ & $1 \%$ & $2 \%$ & $4 \%$ & $6 \%$ & $1 \%$ & $1 \%$ & $0 \%$ & $0 \%$ & $0 \%$ & $1 \%$ & $1 \%$ \\
\hline $100-200$ & $4 \%$ & $2 \%$ & $4 \%$ & $6 \%$ & $4 \%$ & $4 \%$ & $0 \%$ & $0 \%$ & $6 \%$ & $4 \%$ & $6 \%$ \\
\hline $200-300$ & $0 \%$ & $0 \%$ & $0 \%$ & $0 \%$ & $0 \%$ & $0 \%$ & $1 \%$ & $1 \%$ & $1 \%$ & $0 \%$ & $2 \%$ \\
\hline$>300$ & $2 \%$ & $2 \%$ & $6 \%$ & $7 \%$ & $2 \%$ & $2 \%$ & $6 \%$ & $9 \%$ & $4 \%$ & $0 \%$ & $0 \%$ \\
\hline
\end{tabular}

Electrical impedances (Z) of electroencephalography, electrooculography and electromyography electrodes in the recordings with the new designs of the ambulatory electrode set in stage 1 and stage 2 of the study. Data are expressed as percentage of data points within a corresponding range of impedance values. Data points have been collected pointwise (60 s average) from the beginning of the sleep analysis and every full hour after that in all recordings. from the beginning of the recording presumably as the result of inadequate preparation of skin or electrode misplacement.

Recorded signals included clearly recognizable events of different subtypes of SB with concomitant teeth-grinding and arousal events (Fig. 2). There were no significant differences in the perceived ease of applying AES between stage 1 and stage 2 (Table VI). On the average, all of the parts of AES were considered easy to apply.

\section{Discussion}

The aim of this study was to quantify the reliability of the self-applied home PSG recordings conducted with a newly designed AES in subjects with self-reported SB. The failure rate of unattended homePSG with AES was $5 \%$. It is comparable or even lower to failure rates of unattended type 2 home-PSG, in which electrodes are applied by a medical professional (4-8\% [30]-[33] and $20 \%$ [34]). Self-applicability of AES seems to be reliable, since only one recording failed due to problems in applying the AES. However, relatively high number of recordings $(37 \%$ and $24 \%$ in stages 1 and 2 respectively) had at least one electrode with initial bad contact causing signal losses from the beginning of the recording. This was caused mostly by the fact that the size of the electrode sets 


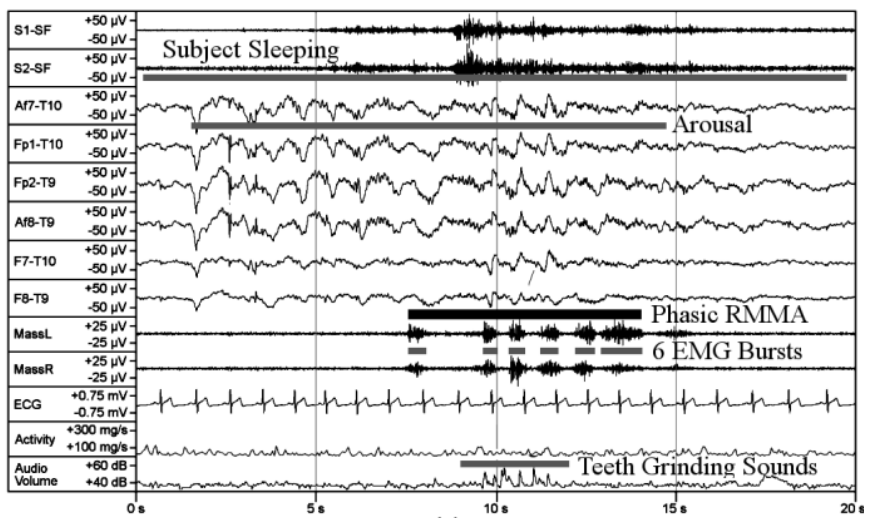

(a)

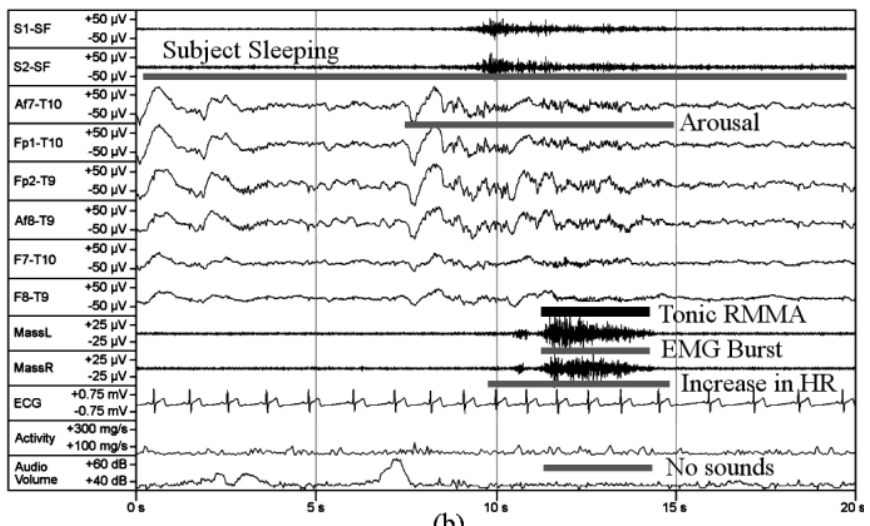

(b)

Fig. 3. Two 20-second samples of recorded signals with ambulatory electrode set (AES) in home setting. AES enables recording of submental electromyography (sub-EMG, channels S1-SF and S2-SF), electroencephalography (channels Af7-T10, Fp1-T10, Fp2-T9 and Af8-T9), electrooculography (channels F7-T10 and F8-T9), bipolar masseter electromyography (mass-EMG, channels MassL and MassR) and electrocardiography (ECG). Nocturnal activity and audio were also recorded. Distinction between (a) phasic (with 6 bursts) and (b) tonic rhythmic masticatory muscle (RMMA) activity could easily be made based on the clear signals on MassL and MassR channels. RMMA events were distinguished from other orofacial or muscular activity and awake bruxism with the help of audio channel and sleep staging according to the flowchart in Fig. 2. An arousal event precedes RMMA events in (a) and (b), in addition to a clear increase of heart rate (HR) in (b), something which could be utilized for automatic recognition of RMMA events.

was fixed and it had to be applied too close to hairline in subjects with narrower faces. In the future, the scalability of the electrode set size should be utilized in the production process to make different sized electrode sets that fit different sized faces better. Another solution would be to use stretchable materials instead of nonstretchable polyester films as the base for the electrode sets [35].

Signal losses were generally restricted to maximum of a few channels at the time when total signal losses caused by recording device malfunction or erroneous set up were excluded. In a recent unattended home PSG study, Bruyneel et al. [33] had more than $95 \%$ of interpretable data (quality grade "A") in both EEG channels in $84 \%$ of the recordings when type 2 PSG equipment for obstructive sleep apnea diagnostics were fitted at home by a technician. In this study this was $84 \%$ and $81 \%$ for any EEG channel and $42-79 \%$ and $52-76 \%$ for single EEG channels (in stages 1 and 2, respectively). Bruyneel et al. [33] suggested that the risk for EEG data loss is not decreased with higher number of EEG channels (four channels instead of two). In contrast to the
TABLE VI

PERCEPTION OF EASINESS TO APPLY THE EQUIPMENT

\begin{tabular}{llll}
\hline \hline $\begin{array}{c}\text { Ease of applying the electrode set and } \\
\text { other devices (1=easy, 10 =difficult) }\end{array}$ & Stage 1 & Stage 2 & \multicolumn{1}{c}{$\begin{array}{c}p \\
\text { value }\end{array}$} \\
\hline EEG, EOG and mass-EMG electrodes & $3.9 \pm 2.4$ & $3.3 \pm 2.0$ & $0.44^{1}$ \\
Sub-EMG electrodes* & - & $2.1 \pm 1.4$ & - \\
ECG electrodes* & - & $2.0 \pm 1.9$ & - \\
Other sensors & $1.8 \pm 1.0$ & $2.0 \pm 1.7$ & $0.61^{1}$ \\
Cables & $1.7 \pm 0.9$ & $1.9 \pm 1.2$ & $0.96^{1}$ \\
\hline
\end{tabular}

The perceived ease of applying the first (Stage $1, \mathrm{n}=19$ ) and second (Stage $2, \mathrm{n}=21$ ) designs of newly designed ambulatory electrode sets and other polysomnography (PSG) sensors (mean $\pm \mathrm{SD}$ ).

1 = Mann-Whitney U-test, $*=$ Included only in the questionnaires in recordings made in stage $2 . \quad \mathrm{EEG}=$ electroenchephalography, $\mathrm{EOG}=$ electroocuclography, mass-EMG = masseter electromyography, sub-EMG = submental electromyography.

study of Bruyneel et al., we found that a high number of EEG channels was beneficial for the success of patient-applied EEG in unattended home PSG.

The AES- 2 design in stage 2 imposed a problem in the cable of sub-EMG electrodes that easily became detached, causing significant data losses in total of five recordings graded "good" or "fair" (Table II). This problem could be solved by introducing wireless data transfer or more securely connected cables between AES and recording device in future development stages. Wireless data transfer would be preferable as it would also reduce noise and movement artifacts, make the AES more comfortable to use, and simplify the self-applicability.

A significant amount of sweat artifact [36] was present in EEG and EOG channels in recordings made with both AES designs. In order to alleviate it AES-2 was designed to cover less skin than AES-1 for improved heat transfer from skin. However, the artifact still persisted and its presence actually increased in recordings made with AES-2. Sweating as the source of the artifact cannot be fully removed, since sweating also depends on the temperature of the sleeping environment and sweating tendency of the subject. Sweating artifact could be removed with high-pass filtering but this would affect the recognition of slow delta waves in N3 sleep stages negatively.

Some of the reasons for signals being uninterpretable were unexpected. Errors in setting up the recording device led to 8.1 $\%$ of total recordings time being lost in stage 2 in all channels (wrong recording time). Most of these signal losses happened when the dentist, unfamiliar with used PSG equipment had set up the device to stop recording earlier than a subject woke up, but it also happened twice for an experienced technician in KUH. This clearly shows the need for recording devices with simple setups if unattended home PSG studies are to be conducted in smaller medical facilities, where the medical personnel is not familiar with the devices. In addition, subjects from UEF and KUH presumably were highly educated (often in health sciences), whereas subjects from the dental clinic had presumably varying educational background. These factors may have a significant role to play on the success rate of AES appliance, since the only failed recording caused by misapplied AES and the majority (76.3\%) of all signal data losses in stage 2 recordings occurred in recordings made in dental clinic.

The measured electrical impedances in home environment are generally in line with and mostly lower compared to those 
reported in our earlier study in lab environment [10]: (44.6 \pm $8.0) \mathrm{k} \Omega$ at $30 \mathrm{~Hz}$ and $(53.1 \pm 8.6) \mathrm{k} \Omega$ at $15 \mathrm{~Hz}$ on skin prepared only by wiping with an ethanol soaked cotton pad. Electrical impedances in this range have a negligible impact on signal quality when modern amplifiers with high input impedances are used [37]. This shows that the electrode contacts and skin impedances are most of the time adequate even when subjects self-apply the electrodes and perform a skin preparation in a simple manner by themselves.

This study had limitations in the small study population and in the lack of comparative concomitant PSG recordings with standardly postitioned electrodes. However, in this study we were especially interested in the success rate and technical quality of PSG recordings where subjects applied the electrodes by themselves. Since standard cup electrodes would have needed to be applied by a technician anyway, we thought that it would have interfered with the testing of selfapplicability. Furthermore, we felt that making comparative non-concomitant recordings was not necessary, as the approximate comparison of technical quality of AES could be made to type 2 PSG technical quality studies made by others [30]-[34]. The results of this study could not be generalized to all sleep disorders and patient groups and the technical quality of AES in the diagnostics of other sleep disorders with differing equipment should be investigated separately.

To conclude, in order to fully utilize the potential of the selfapplicable AES in home PSG, further research is needed on the following topics:

1) Development of different sized or stretchable electrodes.

2) Development of even more reliably attaching electrodes that are easier to apply to reduce the number of channels needed.

3) Implementation of wireless data transfer.

4) Development of simpler devices and softwares to ensure the high technical quality of the recordings in varying user environments.

5) Confirming the diagnostic accuracy of AES for SB in a large validation study in comparison to sleep lab PSG.

6) Development of easily applicable occipital electrodes that would produce good quality signals even on hairy areas, enabling more accurate N1 sleep stage scoring.

7) Development of automatic RMMA detection techniques.

\section{CONCLUSIONS}

AES showed good reliability in unattended home PSG as well as easy and secure self-applicability by subjects. This study demonstrated the benefits of multi-channel measurements in unattended home PSG, when subjects apply the equipment themselves. Proportion of recordings with high quality data from EEG channels of AES were found to be comparable to that of conventional home PSG. In the future, easily applicable AES may offer more accuracy and availability to diagnostics of SB and other sleep disorders such as sleep apnea that requires home PSG but are often currently lacking EEG for sleep staging. If automatic analysis methods are implemented, it should be done in accordance to the fact that single channels of AES may have high proportions of signal quality problems.

\section{ACKNOWLEDGMENT}

The authors would like to thank the staff of Department of Clinical Neurophysiology in Diagnostic Imaging Center of Kuopio University Hospital, Unit of Specialized Oral Care in the Metropolitan Area and Kirkkonummi, Oivauni Oy and Resmed Finland Oy for their contributions to this work.

\section{REFERENCES}

[1] J. E. Ferrie, M. Kumari, P. Salo, A. Singh-Manoux, and M. Kivimäki, "Sleep epidemiology--a rapidly growing field.," Int. J. Epidemiol., vol. 40, no. 6, pp. 1431-7, Dec. 2011.

[2] S. Stranges, W. Tigbe, F. X. Gómez-Olivé, M. Thorogood, and N.-B. Kandala, "Sleep problems: an emerging global epidemic? Findings from the INDEPTH WHO-SAGE study among more than 40,000 older adults from 8 countries across Africa and Asia.," Sleep, vol. 35, no. 8, pp. 1173-81, Aug. 2012.

[3] D. R. Hillman, A. S. Murphy, and L. Pezzullo, "The economic cost of sleep disorders.," Sleep, vol. 29, no. 3, pp. 299-305, Mar. 2006.

[4] J. L. Hossain and C. M. Shapiro, "The prevalence, cost implications, and management of sleep disorders: an overview.," Sleep Breath., vol. 6, no. 2, pp. 85-102, Jun. 2002.

[5] C. A. Kushida et al., "Practice parameters for the indications for polysomnography and related procedures: an update for 2005.," Sleep, vol. 28, no. 4, pp. 499-521, Apr. 2005.

[6] M. Bruyneel and V. Ninane, "Unattended home-based polysomnography for sleep disordered breathing: current concepts and perspectives.," Sleep Med. Rev., vol. 18, no. 4, pp. 341-7, Aug. 2014.

[7] H. Griessenberger, D. P. J. Heib, A. B. Kunz, K. Hoedlmoser, and M. Schabus, "Assessment of a wireless headband for automatic sleep scoring.," Sleep Breath., vol. 17, no. 2, pp. 747-52, May 2013.

[8] J. R. Shambroom, S. E. Fábregas, and J. Johnstone, "Validation of an automated wireless system to monitor sleep in healthy adults.," J. Sleep Res., vol. 21, no. 2, pp. 221-30, Apr. 2012.

[9] B. P. Lucey et al., "Comparison of a single-channel EEG sleep study to polysomnography.," J. Sleep Res., vol. 25, no. 6, pp. 625-635, Dec. 2016.

[10] P. Lepola et al., "Screen-printed EEG electrode set for emergency use," Sensors Actuators A Phys., vol. 213, pp. 19-26, Jul. 2014.

[11] A. Muraja-Murro et al., "Forehead EEG electrode set versus full-head scalp EEG in 100 patients with altered mental state.," Epilepsy Behav., vol. 49, pp. 245-9, Aug. 2015.

[12] S. Myllymaa et al., "Assessment of the suitability of using a forehead EEG electrode set and chin EMG electrodes for sleep staging in polysomnography.," J. Sleep Res., vol. 25, no. 6, pp. 636-645, Dec. 2016.

[13] T. Miettinen et al., "Screen-printed ambulatory electrode set enables accurate diagnostics of sleep bruxism.," J. Sleep Res., May 2017.

[14] F. Lobbezoo et al., "Bruxism defined and graded: an international consensus.," J. Oral Rehabil., vol. 40, no. 1, pp. 2-4, Jan. 2013.

[15] M. C. Carra, N. Huynh, and G. Lavigne, "Sleep bruxism: a comprehensive overview for the dental clinician interested in sleep medicine.," Dent. Clin. North Am., vol. 56, no. 2, pp. 387-413, Apr. 2012.

[16] R. B. Berry, R. Brooks, C. E. Gamaldo, S. M. Harding, C. L. Marcus, and B. V Vaughn, The AASM manual for the scoring of sleep and associated events, 2nd editon. American Academy of Sleep Medicine, 2012.

[17] Y. Yoshida et al., "Association between patterns of jaw motor activity during sleep and clinical signs and symptoms of sleep bruxism," J. Sleep Res., Dec. 2016.

[18] M. C. Carra, N. Huynh, and G. J. Lavigne, "Diagnostic accuracy of sleep bruxism scoring in absence of audio-video recording: a pilot study.," Sleep Breath., vol. 19, no. 1, pp. 183-90, Mar. 2015.

[19] K. M. C. Dutra, F. J. Pereira, P. H. Rompré, N. Huynh, N. Fleming, and G. J. Lavigne, "Oro-facial activities in sleep bruxism patients and in normal subjects: a controlled polygraphic and audio-video study.," $J$. Oral Rehabil., vol. 36, no. 2, pp. 86-92, Feb. 2009.

[20] T. Shochat et al., "Validation of the BiteStrip screener for sleep bruxism.," Oral Surg. Oral Med. Oral Pathol. Oral Radiol. Endod., vol. 104, no. 3, pp. e32-9, Sep. 2007.

[21] F. Jadidi, E. Castrillon, and P. Svensson, "Effect of conditioning 
electrical stimuli on temporalis electromyographic activity during sleep.," J. Oral Rehabil., vol. 35, no. 3, pp. 171-83, Mar. 2008.

[22] K. Ahlberg et al., "Bruxism and sleep efficiency measured at home with wireless devices.," J. Oral Rehabil., vol. 35, no. 8, pp. 567-71, Aug. 2008.

[23] T. Mizumori, S. Inano, M. Sumiya, Y. Kobayashi, T. Watamoto, and H. Yatani, "Ambulatory bruxism recording system with sleep-stage analyzing function.," J. Prosthodont. Res., vol. 53, no. 3, pp. 150-4, Jul. 2009.

[24] T. Yamaguchi, S. Abe, P. H. Rompré, C. Manzini, and G. J. Lavigne, "Comparison of ambulatory and polysomnographic recording of jaw muscle activity during sleep in normal subjects.," J. Oral Rehabil., vol. 39, no. 1, pp. 2-10, Jan. 2012.

[25] T. Castroflorio, A. Deregibus, A. Bargellini, C. Debernardi, and D. Manfredini, "Detection of sleep bruxism: comparison between an electromyographic and electrocardiographic portable holter and polysomnography.," J. Oral Rehabil., vol. 41, no. 3, pp. 163-9, Mar. 2014.

[26] J. Stuginski-Barbosa, A. L. Porporatti, Y. M. Costa, P. Svensson, and P. C. R. Conti, "Diagnostic validity of the use of a portable single-channel electromyography device for sleep bruxism.," Sleep Breath., vol. 20, no. 2, pp. 695-702, May 2016.

[27] T. Kato, P. Rompré, J. Y. Montplaisir, B. J. Sessle, and G. J. Lavigne, "Sleep bruxism: an oromotor activity secondary to micro-arousal.," $J$. Dent. Res., vol. 80, no. 10, pp. 1940-4, Oct. 2001.

[28] P. Anderer et al., "Artifact processing in computerized analysis of sleep EEG - a review.," Neuropsychobiology, vol. 40, no. 3, pp. 150-7, Sep. 1999.

[29] S. Redline et al., "Methods for obtaining and analyzing unattended polysomnography data for a multicenter study. Sleep Heart Health Research Group.," Sleep, vol. 21, no. 7, pp. 759-67, Nov. 1998.

[30] J. M. Fry, M. A. DiPhillipo, K. Curran, R. Goldberg, and A. S. Baran, "Full polysomnography in the home.," Sleep, vol. 21, no. 6, pp. 635-42, Sep. 1998.

[31] C. Iber et al., "Polysomnography performed in the unattended home versus the attended laboratory setting--Sleep Heart Health Study methodology.," Sleep, vol. 27, no. 3, pp. 536-40, May 2004.

[32] M. Bruyneel et al., "Sleep efficiency during sleep studies: results of a prospective study comparing home-based and in-hospital polysomnography.," J. Sleep Res., vol. 20, no. 1 Pt 2, pp. 201-6, Mar. 2011.

[33] M. Bruyneel, W. Libert, L. Ameye, and V. Ninane, "Comparison between home and hospital set-up for unattended home-based polysomnography: a prospective randomized study.," Sleep Med., vol. 16, no. 11, pp. 1434-8, Nov. 2015.

[34] F. Portier et al., "Evaluation of home versus laboratory polysomnography in the diagnosis of sleep apnea syndrome.," Am. J. Respir. Crit. Care Med., vol. 162, no. 3 Pt 1, pp. 814-8, Sep. 2000.

[35] S. Yoshimoto et al., "Wireless EEG patch sensor on forehead using ondemand stretchable electrode sheet and electrode-tissue impedance scanner.," Conf. Proc. ... Annu. Int. Conf. IEEE Eng. Med. Biol. Soc. IEEE Eng. Med. Biol. Soc. Annu. Conf., vol. 2016, pp. 6286-6289, Aug. 2016.

[36] W. O. Tatum, B. A. Dworetzky, and D. L. Schomer, "Artifact and recording concepts in EEG.," J. Clin. Neurophysiol., vol. 28, no. 3, pp. 252-63, Jun. 2011.

[37] T. C. Ferree, P. Luu, G. S. Russell, and D. M. Tucker, "Scalp electrode impedance, infection risk, and EEG data quality.," Clin. Neurophysiol., vol. 112, no. 3, pp. 536-44, Mar. 2001. 